\title{
A novel anti-cancer effect of atelocollagen-conjugated miR-520d-5p on pancreatic cancer cells in vitro and in a mouse xenograft model
}

\author{
Yoshitaka Ishihara $^{1}$, Yugo Miura ${ }^{2,3}$, Norimasa Miura ${ }^{3,4}$ and Keigo Miura ${ }^{3 *}$ \\ ${ }^{1}$ Division of Pharmacotherapeutics, Department of Pathophysiological and Therapeutic Science, Faculty of Medicine, Tottori University, 86 Nishicho, Yonago, \\ Tottori 683-8503, Japan \\ ${ }^{2}$ Department of Orthopedic Surgery, Soka Municipal Hospital, 2-21-1 Soka, Soka, Saitama 340-8560, Japan \\ ${ }^{3}$ PEZY-Pharma, 86 Nishicho, Yonago, Tottori 683-8503, Japan \\ ${ }_{4}$ i-Medical Clinic, 3-4-18 Mejiro, Toshima-ku, Tokyo 171-0031, Japan
}

\begin{abstract}
Background: We have previously shown that hsa-miR-520d-5p can convert undifferentiated hepatoma cells into the status of pluripotent stem cells or mesenchymal stem cells (MSCs) via a demethylation process and P53 upregulation in vivo. Here, we examined anti-cancer effect of miR-520d-5p on three human pancreatic cancer (PC) cells.

Material and methods: After generating GFP-expressing cancer cells, we transfected 520d-5p into three PC cell lines (KLM-1, PK-45p or PK-9) to examine the key gene expression and the change of malignant properties. Also, we examined the therapeutic effect of 520d-5p on KLM- 1 using atelocollagen-conjugated miR$520 \mathrm{~d}-5 \mathrm{p}$ (520d/atelocollagen).

Results: Tumor growth was suppressed by more than $80 \%$ each week by $520 \mathrm{~d} /$ atelocollagen, compared with scramble/atelocollagen (scram./.atelocollagen) or atelocollagen alone. Interestingly, 520d-5p induced PK-9 cells to the transformation accompanied by apoptosis, resulting in the benignant transformation like pancreatic acinar cells with the functions of secreting pancreatic enzyme (lipase or amylase) without malignant properties.

Conclusions: This is the first reports that we confirmed an anti-cancer effect of 520d/atelocollagen or the benignant conversion on PC cells in vivo study. The development to optimize this carrier for drug delivery system (DDS) is expected to elevate the efficiency to enter each tumor cell and provide for the miRNA medicine as a novel therapeutic modality.
\end{abstract}

\section{Introduction}

Mature miRNAs are endogeneous, small, noncoding RNA (ncRNA) molecules 18-23 nucleotides in length that are transcribed by RNA polymerase II and function as post-transcriptional gene regulators, resulting in controlling a gene expression in many cellular processes $[1,2]$ or mediating cellular differentiation, reprogramming and the initiation and progression of human cancer [3-5]. The alteration of miRNA expression levels influences tumor growth by modulating the functional expression of the targeted genes implicated in the regulation of tumor cell apoptosis or proliferation [6]. Central play of miRNAs during tumorigenesis have been investigated in different tumor types, including melanomas, hepatomas, brain tumors, breast tumors and so on [7-9].

We previously identified an RNA gene (RGM249) that may indirectly regulate hTERT (human telomerase reverse transcriptase) expression in undifferentiated cancer cell lines via the induction of double-stranded (ds), small interfering (si), and short hairpin (sh) RNAs. The regulatory function of this gene seemed to be implicated in cell development, cell differentiation, anti-inflammatory effects, and cell growth in undifferentiated cancers [10]. Our report showed that three siRNAs for a kind of small RNAs derived from the ncRNA in vivo affected their metastatic or proliferative abilities during in vivo tumor growth [11].

Ribonucleic acid-based therapeutics have been developed for the application in cancer treatment. For example, viral vectors, nonviral reagents, or nanoparticles have been preclinically or clinically tested to get over the issues such as the instability in vivo, the efficiency, and the inaccurate targeting for organs or tumor tissues [12-18].

We have also reported the safety, efficacy, and specificity of drug delivery systems (DDSs) by utilizing gelatin hydrogel microspheres or atelocollagen for subcutaneous (s.c.) injection and sperminepullulan or atelocollagen for intravenous (i.v.) administration [19-23]. We therefore elucidated the physiological functions of miRNA-like molecules generated from RGM249 and their roles in carcinogenesis,

*Correspondence to: Keigo Miura, PEZY-Pharma, 86 Nishicho, Yonago, Tottori 683-8503, Japan, Tel: 810353488027; E-mail: pezypharmainc@gmail.com

Key words: pancreatic cancer ( $p c)$, atelocollagen, mir-520d-5p, xenograft model, reprogramming

Received: April 01, 2019; Accepted: April 19, 2019; Published: April 26, 2019 
differentiation, and pluripotency and investigate their potential utility for antitumor therapy or regenerative medicine in vivo.

Targeting by miRNA is carried out via base-pair interactions between the 5' end of miRNAs and sites within the coding and/ or untranslated regions (UTRs) of transcriptional form of genes targeted by miRNAs; target sites in the 3' UTR lead to more effective translational dysfunction $[24,25]$. Because one miRNA generally targets at least hundreds of mRNAs, it is considered to be extremely difficult to elucidate miRNA regulatory pathways [26]. In the case of miR-520d-5p that can convert undifferentiated hepatoma cells to benign or normal status in vivo [27], there are more than 1,000 predicted target genes, according to available bioinformatics. Because the effect of miR-520d$5 \mathrm{p}$ on not only cancer cells but also normal cells clarified no toxicity and no tumorigenicity in vivo studies [28,29], we, hereby, examined anticancer effects including the therapeutic effects of $520 \mathrm{~d}-5 \mathrm{p}$ conjugated with an atelocollagen as a DDS carrier on three differentiated types of PC cell lines, by in vivo studies.

\section{Material and methods}

\section{Atelocollagen}

Atelocollagen is a highly purified type I collagen of calf der-mis with pepsin treatment (KOKEN Co., Ltd, Tokyo, Japan).

\section{RNA preparation}

Synthetic 20-nt RNAs were purchased from KOKEN in deprotected, desalted and annealed form. The sequence of our prepared hsa-miR-520d-5p was 5'-cuacaaagggaagcccuuuc-3'; 3'-uugauguuucccuucgggaaag-5'. Non-specific control miRNA duplex was also purchased from HSS (Sapporo, Japan) and the scramble sequence was 5'-gaguccgccucuauagacaa-3'.

\section{Formation of miRNA/atelocollagen complex}

The miRNAs and atelocollagen complexes were prepared as follows according to manufacturer's instructions (KOKEN). Briefly, equal volumes of $1.0 \%$ of atelocollagen [in phosphate-buffered saline (PBS) at $\mathrm{pH}$ 7.4] and miRNAs solution (in PBS) were combined and mixed by rotation $(4 \mathrm{rpm})$ at $4^{\circ} \mathrm{C}$ for $20 \mathrm{~min}$ before centrifugation $(10,000$ $\mathrm{rpm})$ at $4^{\circ} \mathrm{C}$ for $1 \mathrm{~min}$. The complex was used for inoculation into immunodeficient mice (KSN/Slc) (Shimizu Laboratory Supplies Co., Ltd., Kyoto, Japan). The final concentration $(0.5 \%)$ of atelocollagen for subcutaneous injection or intraperitoneal injection in vivo was $10 \mu \mathrm{M}$ or $40 \mu \mathrm{M}$, respectively.

\section{Stability of siRNA/atelocollagen complex}

Aliquots of $0.9 \mathrm{mg}$ of miRNAs and $0.5 \%$ atelocollagen complexes were incubated in the presence of $0.1 \mathrm{mg} / \mathrm{ml}$ RNase A (NipponGene, Tokyo, Japan) for $0,5,15,30,45$ and $60 \mathrm{~min}$ at $37^{\circ} \mathrm{C}$. The solutions were then extracted with phenol and phenol/chloroform/isoamyl alcohol (25:24:1). The miRNAs were precipitated with ethanol, separated by $3.5 \%$ agarose gel electrophoresed and visualized by ethidium bromide staining [29].

\section{Cell lines and immunodeficient mice}

Human PC cell lines (undifferentiated type: KLM-1; moderatelymixed type: PK-45p; well-differentiated type: PK-9) were obtained from Tohoku University, respectively. They were maintained in Roswell Park Memorial Institute (RPMI) 1640 medium (WAKO, Tokyo, Japan) with $10 \%$ heat-inactivated fetal bovine serum (FBS) at $37^{\circ} \mathrm{C}$ in a humidified atmosphere of $5 \% \mathrm{CO}_{2}$. A total of $5 \times 10^{7}$ cells were harvested and inoculated into mice subcutaneously, intraperitoneally or intravenously using a 26-gauge needle. Six-week-old immunodeficient mice were inoculated for in vivo study by miR-520d-5p transfection. Immunodeficient KSN/Slc mice were used to examine the antimetastatic effects of miR-520d-5p or the transformation to normal status by miR-520d-5p. The athymic mice were anesthetized by intraperitoneal injection of $100 \mathrm{mg} / \mathrm{kg}$ Nembutal. All animals were housed and fed in the Division of Laboratory Animal Science at Tottori University (Yonago, Japan) under a protocol that was approved by the Japanese Association for Accreditation for Laboratory Animal Care and animal research and handling were performed in strict accordance with the federal Institutional Animal Care and Use Committee guidelines. All experiments reported in this study were approved by an institutional committee (\#13-Y-37, \#18-2-41, \#h27-074) and ARRIVE guidelines have been followed.

In addition, the human mesangial cell line 293FT (Invitrogen Japan K.K., Tokyo, Japan) was used for producing GFP-expressing lentiviral particles. Briefly, 293FT cells were cultured in DMEM supplemented with 10\% FBS, $0.1 \mathrm{mM}$ MEM nonessential amino acids solution, $2 \mathrm{mM}$ L-glutamine and $1 \%$ penicillin/streptomycin. We abbreviated 6 kinds of transfectants (scramble-transfectants or 520d-5p-transfectants for KLM-1, PK-45p or PK-9) as scram./.KLM-1, 520d/KLM-1, scram./.PK45p, 520d/PK-45p, scram./.PK-9 or 520d/PK-9, respectively.

\section{Lentiviral vector construct and reporter gene labeling of tumor cells}

To examine the effects of miR-520d-5p overexpression, we transfected pMIRNA1-miR-520d-5p/GFP (20 mg; System Biosciences, Mountain View, CA, USA) into 293FT cells $\left(5 \times 10^{6}\right.$ cells $/ 10-\mathrm{cm}$ culture dish). To harvest viral particles, the cells were centrifuged at $170,000 \mathrm{~g}$ $\left(120 \mathrm{~min}, 4^{\circ} \mathrm{C}\right)$. The viral pellets were collected and viral copy numbers were measured with a Lenti-XTM qRT-PCR Titration kit (Clontech, Mountain View, CA, USA). For the infection into KLM-1 cells, $1 \mathrm{x}$ $10^{6}$ lentiviral copies were used per $10-\mathrm{cm}$ culture dish. To estimate the efficacy of infection by the GFP-expressing lentiviral vector (pMIRNA1/ GFP: $20 \mu \mathrm{g}$; System Biosciences, Mountain View, CA, USA), GFP expression in PC cells was detected with the EVOS FL cell imaging system. Transfectants without viral particles in the culture supernatant were used for tumor formation in vivo.

\section{Gene expression analysis by reverse transcription-polymerase chain reaction (RT-PCR)}

Total RNA, including the small RNA fraction, was extracted from cultured cells or homogenized mouse tissues with the mirVana miRNA Isolation Kit (Ambion, Austin, TX, USA). Mature miRNA (miR520d-5p: $25 \mathrm{ng} / \mu \mathrm{L}$ ) was quantified with the Mir- $\mathrm{X}^{\mathrm{ix}}$ miRNA qRT-PCR SYBR' kit (Takara Bio, Tokyo, Japan) according to the manufacturer's instructions. The gels were run under the same experimental conditions. PCR and data collection analyses were performed with a BioFlux LineGene (Toyobo, Nagoya, Japan). To analyze the RT-PCR results, all data were normalized to a $\beta$-actin internal control. U6 small nuclear RNA was also used as an internal control. Total RNA $(25 \mathrm{ng} / \mu \mathrm{l})$ was reverse transcribed and amplified using the KAPA SYBR FAST OneStep qRT-PCR Kit (NIPPON Genetics Co, Ltd, Tokyo, Japan). RNA quantification was confirmed by sequencing with high reproducibility. Supplementary Table 1 shows the primer sequences that were used for mRNA or miRNA quantification. The data were analyzed statistically by one-way analysis of variance (ANOVA) or Mann-Whitney $U$ tests, and significant differences are shown as ${ }^{*}: \mathrm{P}<0.05$ and ${ }^{* *}$ : $\mathrm{P}<0.01$. 


\section{Fluorescence detection in cells}

To estimate the efficacy of infection by the hsa-miR-520d-5pexpressing lentiviral vector, GFP expression was detected with an OLYMPUS IX71 microscope using a TH4-100 power supply (Tokyo, Japan).

\section{Cell migration assay}

The invasive abilities of transfected cells (scram./KLM-1 and 520d/ KLM-1, scram./PK-45p and 520d/PK-45p or scram./PK-9 and 520d/ PK-9) were estimated using a CIM-Plate 16, which detects cell invasion/ migration in real time following the manufacturer's instructions (xCELLigence system, Roche, Basel, Switzerland).

\section{Flow cytometry analysis}

Cell cycle analysis was conducted to confirm the DNA content and changes in cell populations in the 520d/KLM-1,520d/PK45p or 520d/ PK-9 cells as previously reported for 520d/HLF (undifferentiated type of hepatoma cell line) [27]. The DNA content of the cells was analyzed with a flow cytometer (Epics Altra; Beckman Coulter Inc., CA, USA). Cells were assessed by approximately 20,000 collected events after the transfection of a pMIRNA1-miR-520d-5p/GFP clone using EXPO32 ADC Analysis software. GFP-positive cells were sorted on a Moflo XDP cell sorter (Epics Altra; Beckman Coulter Inc.). Specifically, for cell cycle analysis, a single cell suspension was washed once with cold PBS. The cell pellet was loosened by shaking the tube gently and was fixed with $3.7 \%$ formalin in $\mathrm{ddH}_{2} \mathrm{O}$, which was added dropwise. The cells were then incubated at least overnight at $22^{\circ} \mathrm{C}$. After fixation, the cells were washed twice with cold PBS to remove the ethanol, and resuspended at $1 \times 10^{6}$ cells $/ \mathrm{ml}$ in PBS with $100 \mathrm{U} / \mathrm{ml}$ RNase A and incubated for $50 \mathrm{~min}$ at $37^{\circ} \mathrm{C}$. Next, $50 \mathrm{mg} / \mathrm{ml}$ of propidium iodide were added, and the mixture was incubated for $40 \mathrm{~min}$ on ice in the dark. The DNA content was analyzed with a flow cytometer; the cells were assessed from approximately 20,000 collected events after the transfection of a pMIRNA1-miR-520d-5p/GFP clone and prior to EXPO32 ADC Analysis.

\section{miRNA transfer procedures in vivo}

The miR-520d-5p/atelocollagen complex was injected into immunodeficient mice topically, intraperitoneally or systemically [29]. To identify the anti-cancer effects of miR-520d, we examined its effects on KLM-1 cells in vivo. Atelocollagen was used as a carrier. The cells were subcutaneously inoculated into the right flanks of the mice $(n=8$ for each cell line), intraperitoneally into the center of the abdomen $(n=8)$ or intravenously into the caudal vein $(n=8)$. Because miRNA expression in tumors was evaluated after categorizing the parts of the tumor as described in our previous report [11], we started examining the therapeutic effect when tumor size was $8 \mathrm{~mm}$ for KLM-1 in a subcutaneous xenograft model. The miRNA/atelocollagen conjugates were administered to 6-week-old mice every 7 days for approximately 100 days. The observation period was a maximum of 6 months for mice whose inoculated tumors disappeared. The animals were sacrificed after 12 weeks for KLM-I and examined for gross tumor formation and metastasis. Mice prepared for systemic delivery received an intravenous injection every week, starting 1 week after the first injection of KLM1 cells. The injection volume was $200 \mu \mathrm{l}$ and comprised $1 \times 10^{7}$ cells. Palpable tumors were confirmed on day 7 following inoculation. Volume estimations were determined using the following formula: volume $=(\pi / 6 \times$ width $\times$ length $\times$ height $)$. After the mice were sacrificed, we assessed the intrahepatic tumors, intraperitoneal dissemination, micrometastases in organs (brain, lung, heart, spleen, small intestine, liver and kidney) and the presence or absence of adverse effects of atelocollagen-related adverse effects, such as intravascular embolism. In an intraperitoneal xenograft model, therapeutic injection was initiated one week later than the inoculation at the same concentration of $520 \mathrm{~d} /$ atelocollagen as of the subcutaneous injection. Via systemic administration through the caudal vein, $0.05 \%$ of $520 \mathrm{~d} /$ atelocollagen was used one week later or earlier than the inoculation. Intratumoral gene expression was polymers estimated according to our previously reported method [11].

\section{Evaluation of miRNA transfer in vivo}

RNA quantification was confirmed by sequencing with high reproducibility. Tissues were subjected to miRNA extraction using a mirVana miRNA Isolation kit, and miRNA expression was examined using a Mir- $\mathrm{X}^{\mathrm{su}}$ miRNA qRT-PCR SYBR kit to confirm the suppression by siRNA and to evaluate changes in miRNA expression using the $2^{-\Delta \Delta \mathrm{Ct}}$ method according to the manufacturer's recommendations. Genes were chosen from those with altered expression levels in response to miR-520d-5p induction and silencing.

\section{Analysis of miRNA delivery using in vivo imaging}

KLM-1 cells were subcutaneously injected $\left(5 \times 10^{7}\right.$ cells per site) into the athymic nude mice after the transfection by scramble or $520 \mathrm{~d}-5 \mathrm{p}$. When the tumor grew to $8 \mathrm{~mm}$ in size, atelocollagen alone, control miRNA conjugated with atelocollagen (scramble), or 520d-5p/atelocollagen was injected around the tumors as described previously [11]. Each group consisted of 8 animals. In vivo bioimaging was conducted on a cryogenically cooled IVIS system (Xenogen Corp., Waltham, MA, USA) using LivingImage acquisition and analysis software [30]. Tumor growth was monitored by measuring the light emission from individual mice 21 to 28 days after miRNA administration. The therapeutic effect of $520 \mathrm{~d}-5 \mathrm{p}$ on subcutaneously inoculated tumors was evaluated based on the intensity of GFP signals by using In vivo imaging system. Tumor growth was not affected by these treatments.

\section{Histological examination}

Tumor nodules were investigated macroscopically or under a dissecting microscope with bright-field imaging. The tissue samples were fixed in $10 \%$ buffered formalin overnight, washed with PBS, transferred to $70 \%$ ethanol, embedded in paraffin, sectioned and stained with hematoxylin-eosin (HE). GFP expression in tissues was observed in unstained, and HE staining samples using an EVOS FL cell imaging system (Life Technologies Japan, Tokyo, Japan).

\section{Statistical analysis}

Mann-Whitney $U$ test was used for comparisons between the controls, the scramble and/or the miR-520d-5p results with one observed variable. P-values considered to indicate a significant difference was labeled as follows: ${ }^{*} \mathrm{P}<0.05$ and ${ }^{* *} \mathrm{P}<0.01$. In box plots, the top and bottom of each box represent the 25 th and 75 th percentiles, respectively, thus providing the interquartile range. The line through the box indicates the median, and the error bars indicate the 5th and 95th percentiles.

\section{Results}

As we reported previously [27], we provided new evidence that HLF cells could be converted to benign or normal status via stemnessmediated process by miR-520d-5p. In this study, we assessed the tumorsuppressive effect on PC cells in vitro and attempted to examine the 
therapeutic effect of the miRNA on three cell lines (KLM-1, PK-45p or PK-9) using atelocollagen as a biomaterial for a DDS carrier although it completely penetrates to all tumor cells comprised of tumor tissues, resulting that viable cells remained more or less. We lenti-virally transfected 520d-5p to PC cells in vitro and confirmed a formation of spheroid-like populations that expressed a reporter gene (GFP) (Figure 1), pluripotent markers Nanog and Oct4, P53, HDAC and CD105 (Figure 1) commonly in transfectants. After we confirmed miR-520d expression in them (Figure 1), they were analyzed DNA content using flow cytometry, resulting that they shifted towards the mixed status of homogeneous proliferation in $S$ phase and apoptotic cell populations in
preG1 phase (Figures 2 and 3). 520d/KLM-1 cells did not pass through fibronectin membrane in migration assay unlike mock/KLM-1 or KLM-1 cells (Figure 3).

We performed in vivo studies using 520d-5p-conjugated atelocollagen (520d/atelocollagen) to assess the topic effects on cancer tissues after we confirmed intense GFP and 520d-5p expression (Figure 4). After inoculating KLM-1 cells into nude mice subcutaneously (Figure 4), 520d/atelocollagen was injected one per week after a tumor becomes $5-8 \mathrm{~mm}$ in size (corresponding to approximately $3-4$ week after the inoculation). Average fluorescence intensity after subcutaneous injection of 520d/atelocollagen were recorded and depicted in Figure 4.
$520 d / K L M-1$
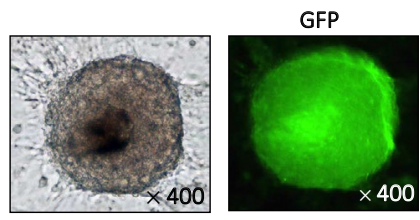

$520 d / P K-45 p$

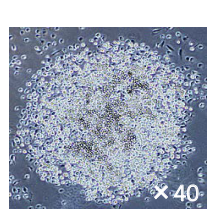

GFP

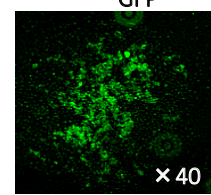

$520 d /$ PK-9

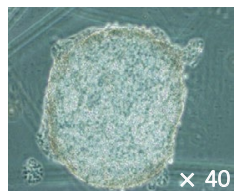

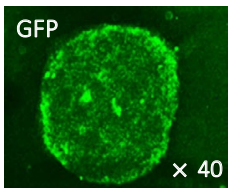
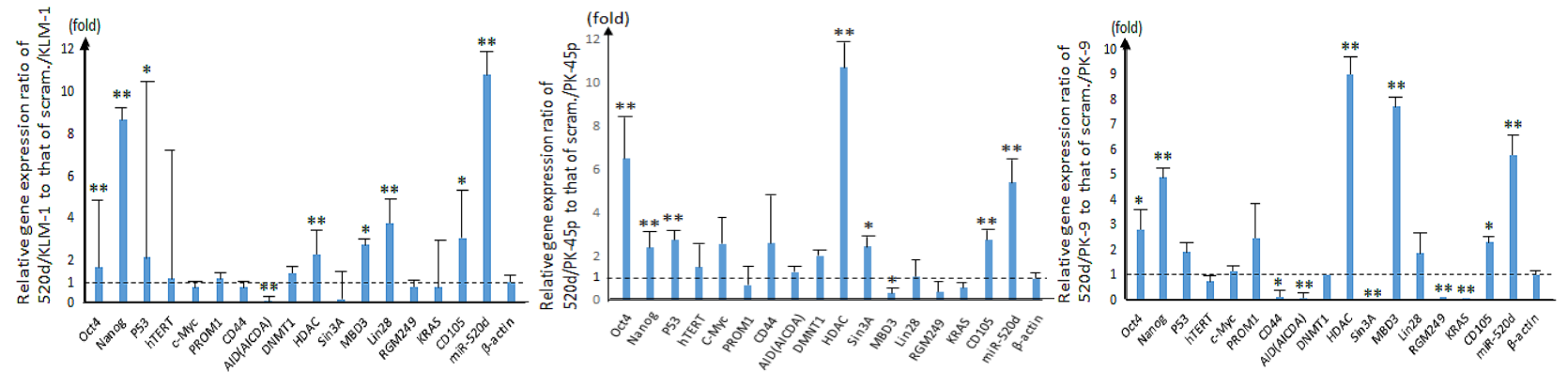

Figure 1. The phenotypes of PC cell lines (520d/KLM-1: left; 520d/PK-45p: middle; 520d/PK-9: right) were representatively shown (x100 magnification). Spheroid-like cells comprise small size of cell populations was shown (top left). By the effective induction (more than $99 \%$ ), we confirmed the GFP expression in the transfectants (x100 magnification) (top right). The phenotypic changes were not sufficient to assess whether they were malignant or benign. Significant 520d-5p expression was confirmed in $520 \mathrm{~d}-$ transfectants $(*$ : $\mathrm{P}<0.01$ by Mann-Whitney $U$ test) (bottom). Relative the genes' expression and miR-520d expression in 520d/KLM-1, 520d/PK-45p or 520d/PK-9 to mock-transfectants were shown, respectively. All expression data were standardized to $\beta$-actin expression level
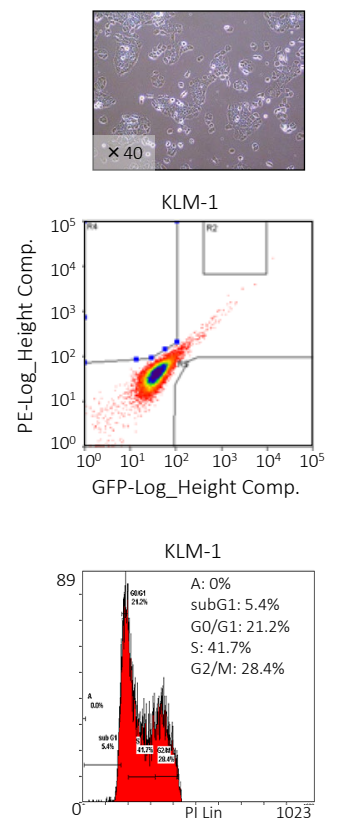
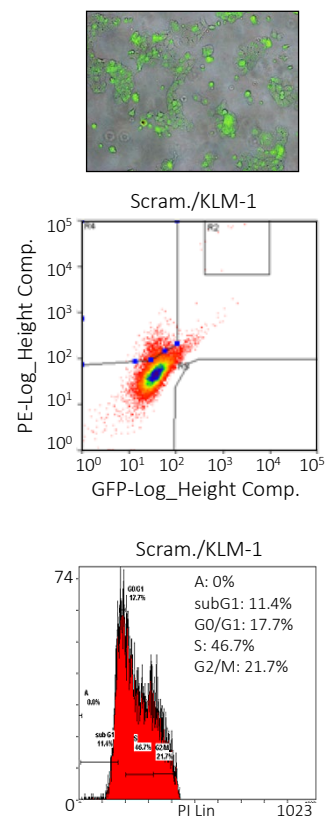
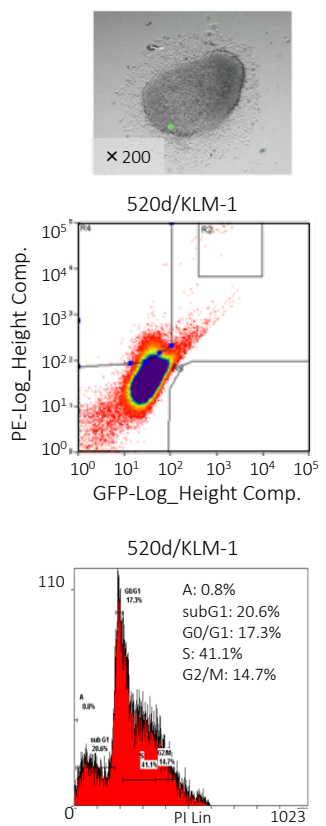

Figure 2. FACS analysis representatively showed that the GFP-positive 520d/KLM-1 cells (top middle) had a higher DNA content in the S phase than the GFP-positive mock/KLM-1 cells (left). 520d/KLM-1 had cell fractions in the preG1 phase that we could not find in mock-transfectants (bottom right) 

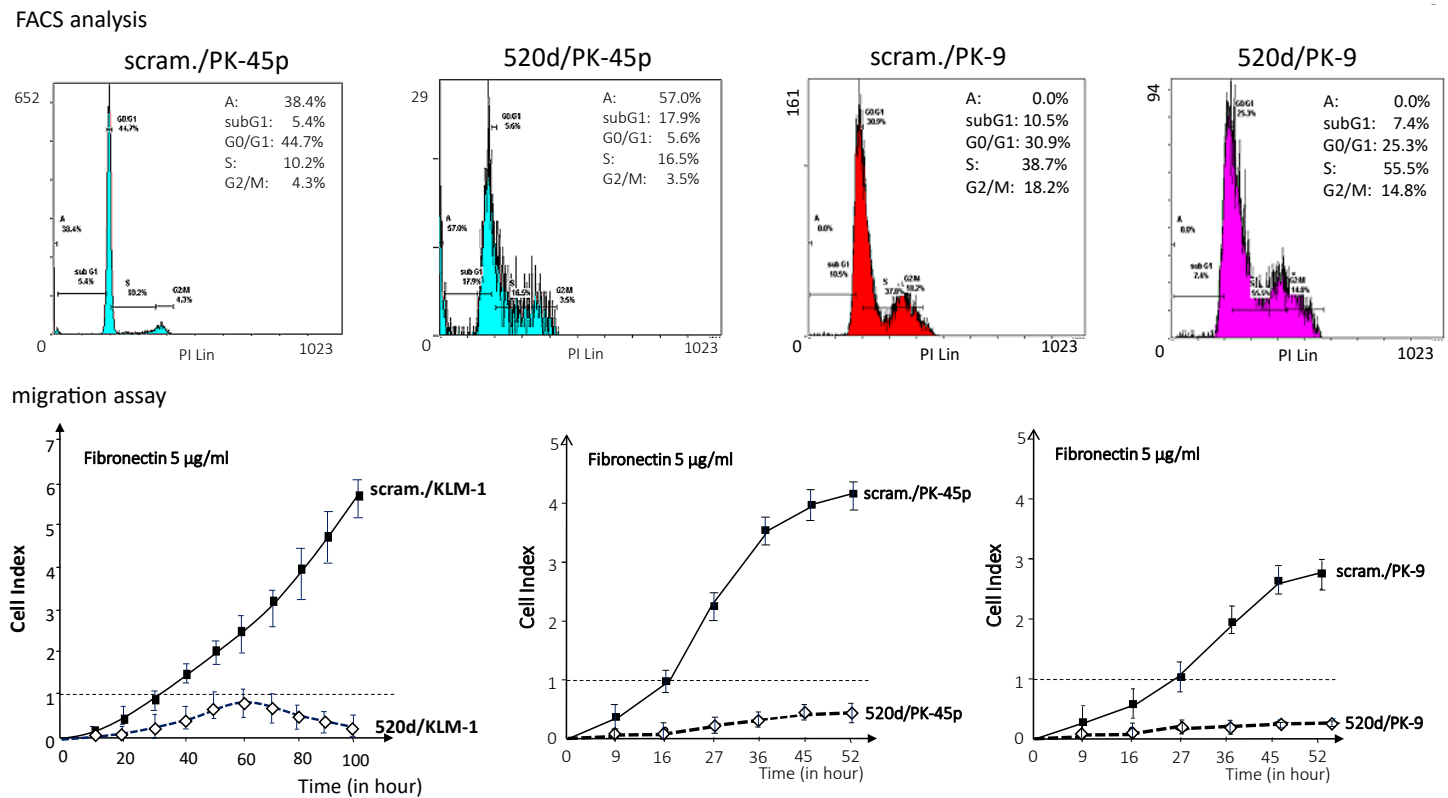

Figure 3. The invasive abilities of KLM-1 and mock-transfected KLM-1 (scram./KLM-1) treated with miR-520d-5p (520d/KLM-1) were estimated in a migration assay, using a fibronectin membrane $(5 \mu \mathrm{g} / \mathrm{ml})$. Most of the $520 \mathrm{~d} / \mathrm{KLM}-1$ cells could not pass through the membrane (bottom left). Similarly, FACS analysis or migration assay was performed in PK-45p (top left or bottom middle, respectively) or PK-9 (top right or bottom right, respectively). 520d/PK-45p or 520d/PK-9 was induced to homogeneous proliferation accompanied with or without apoptotic phase, respectively (top left or top right). Migration assay showed that PK-45p or PK-9 could not pass through the membrane (bottom middle or bottom right)

a

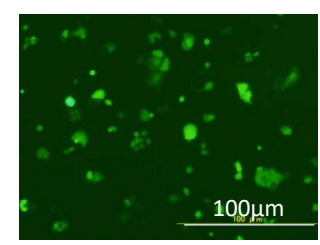

b

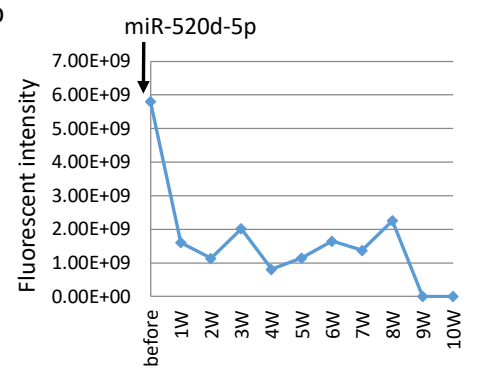

C

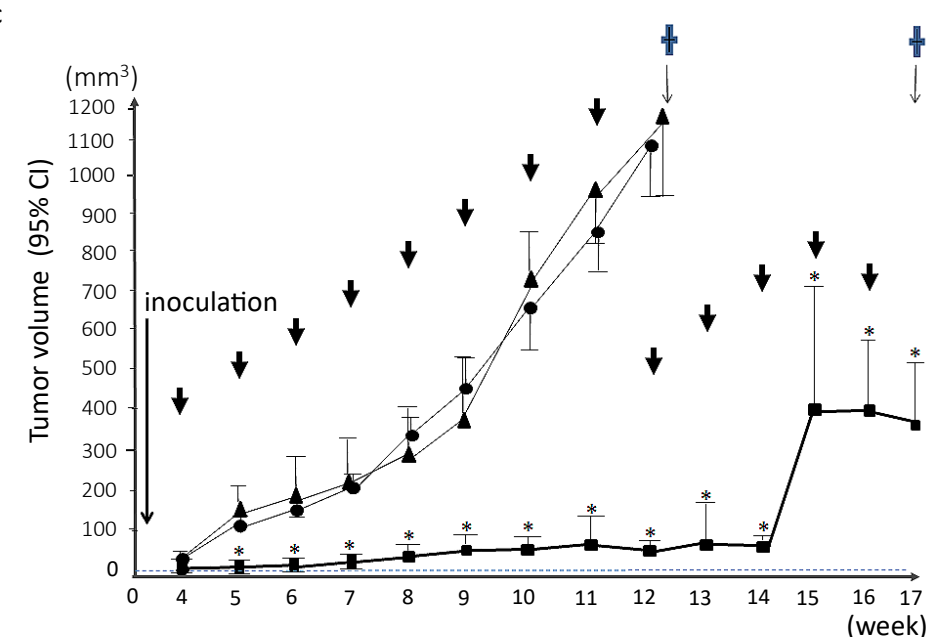

Figure 4. After we generated GFP-expressing KLM-1 (KLM-1/GFP) cells using lentiviral construct, the therapeutic effects was examined by the subsequent injection of 520d/atelocollagen

a. The average fluorescent intensity in tumor was measured and we representatively showed them until the eighth week (bottom for each). GFP expression was maintained during the period of observation. The dotted line indicates tumor growth in control.

b. Overviews of in vivo study using $520 \mathrm{~d}$ /atelocollagen in a xenograft model (bottom). In the group administrated 520d/atelocollagen complex, tumor volume was significantly suppressed compared with that in controls. *: $\mathrm{P}<0.01$ by Mann-Whitney $U$ test. The tumors from $13 \mathrm{~W}$ to $17 \mathrm{~W}$ (sacrificed point) grew rapidly although they received the atelocollagen-conjugated medicine. $\downarrow$ : injection of complex (once a week), $\boldsymbol{\Delta}$ : Atelocollagen alone, $\bullet$ : scramble/atelocollagen, $\mathbf{m}:$ miR-520d-5p/atelocollagen (cases with suppressive growth), $\square$ : miR-520d-5p/ atelocollagen (cases with disappearance of tumor), $\dagger$ : mice were sacrificed, CI: confidence interval 
KLM-1 tumors injected subcutaneously were significantly suppressed until 14 weeks, followed by the rapid growth of a recurrent tumor (Figure 4).

Regarding KLM-1 tumors, average $89.0 \%, 75.0 \%(6 / 8)$ or $100 \%(8 / 8)$ was suppressed in subcutaneous model in tumor size, intraperitoneal model or systemic model (metastasis), respectively (Table 1). Suppressive effect of miR-520d-5p on subcutaneous tumors or metastasis was calculated based on the week data or findings at the sacrifices. $25.0 \%(2 / 8)$ of GFP-expressing KLM-1 cells received 520d$5 p$ (520d/KLM-1/GFP) gradually decreased the signal, following the disappearance in a $5^{\text {th }}$ week, but $75.0 \%(6 / 8)$ of tumors followed the similar reduction of tumors till approximately 14 weeks, followed by the onset of tumor growth (Figures 4 and 5), while GFP-expressing KLM-1 cells received scramble (scramble/KLM-1/GFP) showed an uptake of GFP signal accompanied with a gradual growth (Figure 5). Histological changes were examined after resecting tumors, injection sites or main organs including the vessels. Visible tumors resected at the end of observation period no longer suppressed the growth, we observed that the resected tumors were occupied by white atelocollagen. In addition, adverse effects and toxicity were assessed in this study. For six months when $25 \%(2 / 8)$ of tumor-inoculated mice could be observed, their normal activities were maintained without a weight loss or anorexia and they did not undergo a toxic reaction (such as embolism) by the infusion of atelocollagen.

Additionally, when we injected pancreatic ductal adenocarcinoma cells (PK-9) transfected by 520d-5p intraperitoneally $(n=8)$, we observed no tumor formation $(50.0 \% ; 4 / 8)$, hemorrhagic ascites without any tumors $(25.0 \% ; 2 / 8)$, and benign cyst formation at the injected part of the abdominal wall $(25.0 \% ; 2 / 8)$, although mocktransfected PK-9 generated many tumor nodules in intraperitoneal cavity. Arrows indicate the intraperitoneal nodules (Figure 6) and three phenotypes consisting of no tumor formation (Figure 6), hemorrhagic ascites (Figure 6), and $20 \mathrm{~mm}$ cyst formation (Figure 6) are shown. We examined whether hemorrhagic ascites contained the cancer cells but tumor cells were never detected microscopically. Therefore, we hypothesized that digestive juices derived from pancreatic cells transformed by $520 \mathrm{~d}-5 \mathrm{p}$ were produced and damaged the tissues in the peritoneal cavity. As a result, the fluid included lipase and amylase and, on the other hand, trypsin was not detected although the original cancer cells had produced faintly it. Iode-starch reaction was performed, suggesting that mice \#1 and \#2 faintly included amylase in hemorrhagic ascites and \#3 included amylase in the content of cyst (Figure 6) unlike mice \#4 (Figure 6). In this study, a malignant phenotype could be never seen in transfectants.

\section{Discussion}

A drug delivery carrier using ribonucleic acid-based medicine has undergone repeated improvements. It is urgent issues to develop the formulations or optimize the modification of molecules themselves, due to the instability of RNA molecules when they are handled biotechnically or applied in vivo study to evaluate the usefulness [3133]. Accumulating evidences have suggesting that miRNAs are strongly linked to numerous biological processes including oncogenesis or tumor progression [34-36]. The improvement of nanoparticles [37], cationic polymers [38], antisense [31] or mimic RNA [39], virus [13,40], and

Table 1. The suppression rate (\%) of tumor volume in subcutaneous model, therapeutic effect in peritoneal model, or anti-metastatic effect using the injection from the caudal vein compared with the control in each week is shown in KLM-1 cells. We observed $89 \%$ tumor suppression effect, $75 \%$ therapeutic effect or $100 \%$ anti-metastatic effect

\begin{tabular}{|c|c|c|c|}
\hline & Summary of therapeutic effect miR-520d-5p/atelocollagen complex in a xenograft model \\
\hline & Tumor suppressive effect & Therapeutic effect \\
\hline Cancer cells & $\begin{array}{c}\text { in subcutaneous model }(\mathrm{n}=8 \text { for each) } 8 \\
\text { weeks, average } \%\end{array}$ & $\begin{array}{c}\text { in peritoneal model (n=8 for each) } \\
\text { (effective/total) }\end{array}$ & $\begin{array}{c}\text { in caudal vein model (n=8 for each) } \% \\
(\text { effective/total) }\end{array}$ \\
\hline KLM-1 (undifferentiated PC cells) & 89 & $75.0(6 / 8)$ & $100(8 / 8)$ \\
\hline
\end{tabular}

Effective/total: the number of mice with tumor disappearance/total number of examined mice.

8 weeks, average \%: average of tumor suppressive rate each week compared with control during 8 week-observation.

a

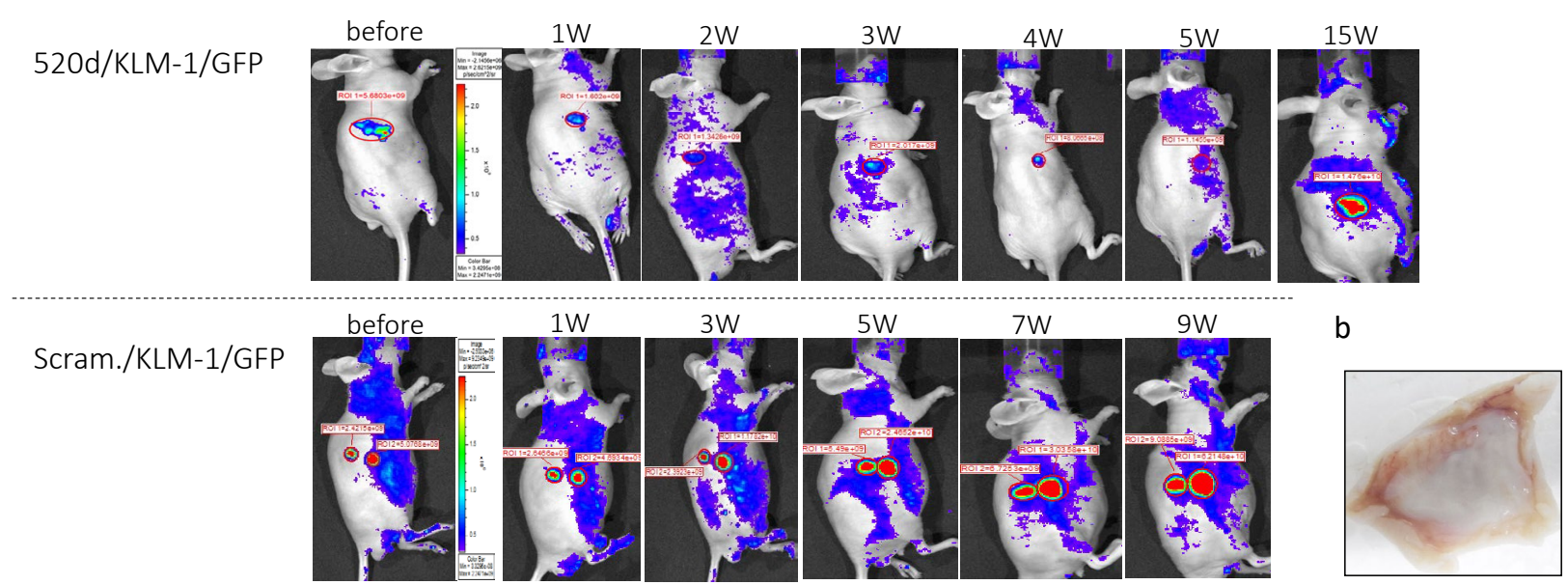

Figure 5. The therapeutic effect of 520d-5p/atelocollagen using in vivo imaging system was representatively shown. $25.0 \%(2 / 8)$ of tumor disappeared in each inoculated site and tumors received 520d-5p were significantly suppressed the growth and the metastatic ability was entirely inhibited other tumors of remaining mice. The time course of $520 \mathrm{~d} / \mathrm{KLM}-1 / \mathrm{GFP}$ cells (520d-5p-transfected and GFP-expressing KLM-1) or scram./KLM-1/GFP cells (scramble-transfected GFP-expressing KLM-1) was depicted (top or bottom, representatively)

a. 1-2 mm protruding but never growing lesions of the no viable tumor

b. The injected site was macroscopically observed after the sacrifice. The lesion was totally occupied with atelocollagen which became white and we did not observe any viable tumor cells microscopically. 
artificial virus [41] has been added energetically for superior quality. Atelocollagen is also a DDS carrier used for topical use in cosmetics or plastic and reconstructive surgery. This biomaterial is extending a possibility for intraperitoneal or systemic administration as well as subcutaneous injection in cancer therapy [23,42-45].

In this study, we first attempted to examine the tumor suppressive effect of miR-520d-5p using atelocollagen by subcutaneous injection. Because this DDS carrier could not penetrate into all cancer cells and viable cancer cells in tumor tissues were left, we used the assay system we established for optimizing the injection condition [29], according to the previous report that atelocollagen accumulates in the grade IV prostatic adenocarcinoma cells [46]. The tumor is suppressed the growth significantly regarding tumor size for each week., as far as we start injecting $520 \mathrm{~d} /$ atelocollagen into subcutaneous tumor at tumor size not to exceed $8 \mathrm{~mm}$ in KLM-1 cells. Upregulation P53 and Nanog, and downregulation of AICDA observed in common with other undifferentiated cancer cells (glioblastoma, PC and anaplastic thyroid cancer) in vitro study could not significantly confirm the intratumoral expression when mice were sacrificed, compared with that in parental or scramble-transfected cells. Although most mice did not sufficiently grow any tumor nodules in their bodies, the most outstanding effect was the suppression of metastasis. Only if when we inject the pharmaceutical not in an intratumoral manner but to wrap up a tumor from around tumor, mice with tumour-inhibiting effect did not provide any (micro)metastatic findings even in the surrounding tissues of tumor. As another outcome we cannot overlook in this study, there may be possible that in PC cells, independently of the differentiation type, apoptosis was observed after transfection of the microRNA, although not apoptosis but transformations of transfectants was observed in undifferentiated type of hepatoma cells (HLF). For the reason we hypothesize P53 upregulation precedes the demethylation process in PC cells (Figure 7). If the demethylation process precedes P53 upregulation, the transfectants were induced mainly to the
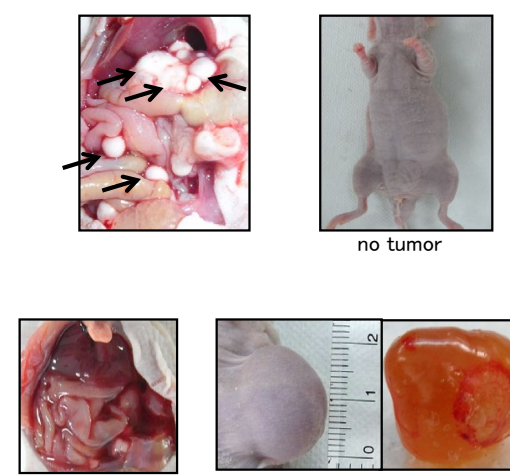

hemorrhagic ascites

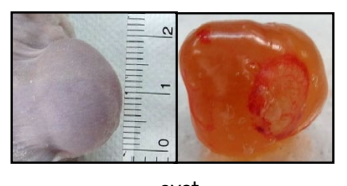

cyst b

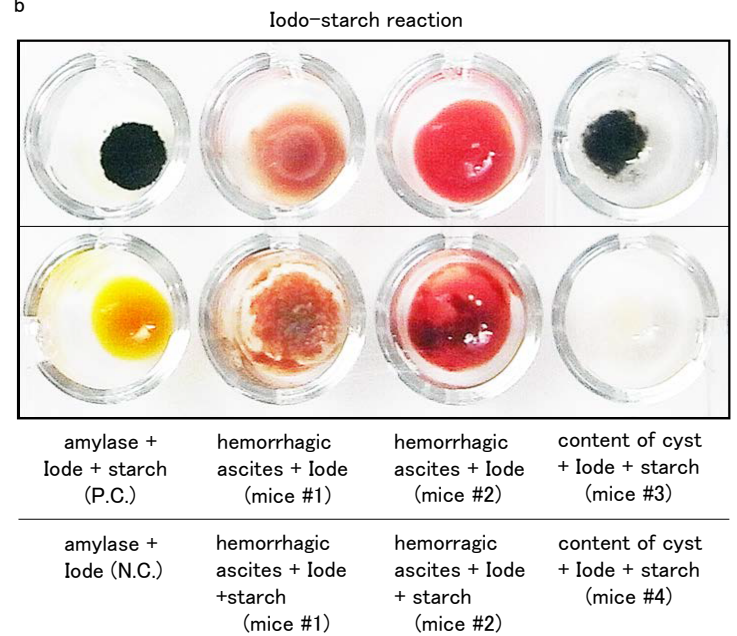

Figure 6. Although mice received scram./PK-9 cells intraperitoneally generated multiple tumor nodules (top left, arrow), 520d/PK-9 cells formed no tumors (top right), induced hemorrhagic ascites (bottom left), or formed $20 \mathrm{~mm}$ cysts (bottom right) in separate individuals. No malignant tumors could be formed

a. As cancer cells were observed in the hemorrhagic ascites contrary to our guess, we examined Iode-starch reaction as a representative of pancreas-related property. The minute amount of amylase except for lipase was detected in the ascites (bottom middle: mice \#1, \#2). The similar amount of amylase to control (top left) was detected in one of cystic tumors (top right: mice \#3)

P53 precedes demethylation

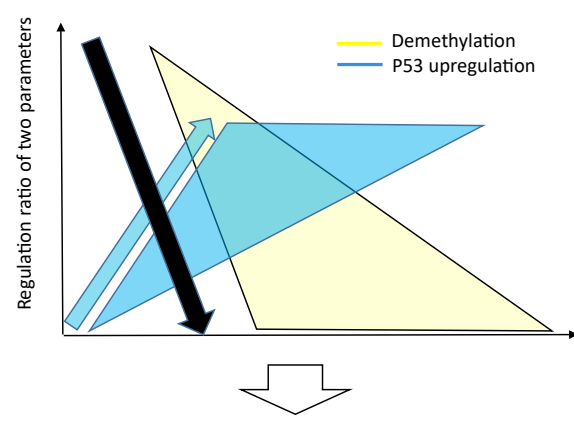

apoptosis
Demethylation precedes P53 upregulation

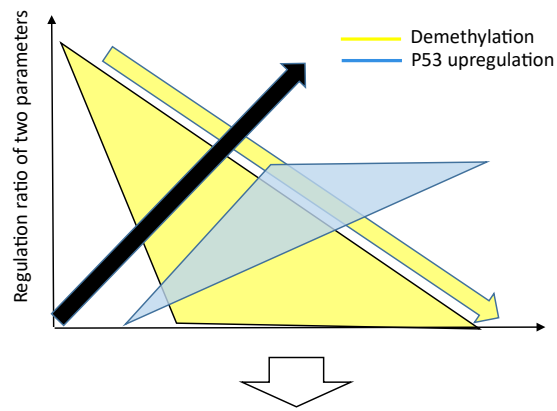

transformation (reprogramming)

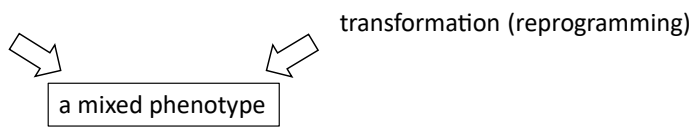

Figure 7. In PC cells, we observed both apoptotic fractions and synthetic proliferation including benign transformations in vivo test. The mixed phenotype may explained by two molecular factors. When P53 upregulation precedes demethylation process, apoptotic induction might be seen mainly. When demethylation process precedes P53 upregulation, reprogramming (the loss of malignancy) might be induced without apoptosis. The black arrow (downward or ascending) indicates the cell death or the surviving status, respectively. The yellow arrow and triangle show demethylation process, the blue arrow and triangle show P53 upregulation process 
transformation, as we reported in HLF previously [27]. Therefore, we presumed which process of the two inductions precedes depended on the molecular reaction of cell types. Furthermore, only if the high efficiency of $520 \mathrm{~d}$ transfection is possible, PC cells may be converted to the loss of malignancy via reprogramming process.

The property that atelocollagen can prevent immune-stimulatory adverse effects [46] and the suppressive effect of immunoreaction may be involved in this phenomenon [27]. In that sense, 520d/atelocollagen as a DDS carrier may be an interesting molecule that the action mechanism and pharmacodynamics should be clarified.

Thus, 520d/atelocollagen might be potent biomaterial as an optional candidate of therapeutic agents. Because the efficiency of the introduction of such conjugating materials into a target cell or a target tissue remains unclear, further and careful investigation will be needed, looking into the application to the human body.

\section{Conclusion}

In conclusion, this study may provide the second evidence that 520d-5p/atelocollagen vehicle is a useful biomaterial for in vivo xenograft model or clinical use towards anti-cancer therapies. Also, $520 \mathrm{~d} /$ atelocollagen can be used as a novel anti-cancer agent that can induce apoptosis as well as transformation.

\section{Acknowledgements}

We thank Dr. Satoshi Kuwamoto and Dr. Junichi Hasegawa for assistance with pathological examination and providing an environment for this study.

\section{Author contributions}

The author(s) have made the following declarations about their contributions: NM conceived and designed the experiments, YI and $\mathrm{KM}$ performed the experiments, $\mathrm{KM}$ analyzed the data, YI and YM contributed reagents/materials/analysis tools. YI, NM and KM wrote the manuscript.

\section{Funding}

This work was supported by a Grant-in-Aid for Research for Promoting Technological Seeds B (development type), the Takeda Science Foundation, the Princess Takamatsu Cancer Research Fund (11-24313), and the Adaptable and Seamless Technology Transfer Program through Target-driven R\&D (Exploratory Research) of the Japan Science and Technology Agency (JST).

\section{Availability of data and materials}

The datasets supporting the conclusions of this article are included within the article. Any request of data and material may be sent to the corresponding author.

\section{Competing interests}

The authors declare that they have no competing interests.

\section{Consent for publication}

Not applicable.

\section{Ethics approval and consent to participate}

All animal experimental protocols were approved by the ethics committee (Institutional Animal Care and Use Committee) of Tottori University.

\section{References}

1. Lagos-Quintana M, Rauhut R, Lendeckel W, Tuschl T (2001) Identification of novel genes coding for small expressed RNAs. Science 294: 853-858. [Crossref]

2. Meltzer PS (2005) Cancer genomics: small RNAs with big impacts. Nature 435: 745 746. [Crossref]

3. Chen CZ, Li L, Lodish HF, Bartel DP (2004) MicroRNAs modulate hematopoietic lineage differentiation. Science 303: 83-86.

4. Thum T, Galuppo P, Wolf C, Fiedler J, Kneitz S, et al. (2007) MicroRNAs in the human heart: a clue to fetal gene reprogramming in heart failure. Circulation 116: 258-267. [Crossref]

5. Viswanathan SR, Daley GQ, Gregory RI (2008) Selective blockade of microRNA processing by Lin28. Science 320: 97-100. [Crossref]

6. Chan JA, Krichevsky AM, Kosik KS (2005) MicroRNA-21 is an antiapoptotic factor in human glioblastoma cells. Cancer Res 65: 6029-6033.

7. Ma L, Weinberg RA (2008) MicroRNAs in malignant progression. Cell Cycle 7: 570572. [Crossref]

8. Meng F, Henson R, Wehbe-Janek H, Smith H, Ueno Y, et al. (2007) The MicroRNA let7a modulates interleukin-6-dependent STAT-3 survival signaling in malignant human cholangiocytes. J Biol Chem 282:8256-8264.

9. Satzger I, Mattern A, Kuettler U, Weinspach D, Voelker B, et al. (2010) MicroRNA$15 \mathrm{~b}$ represents an independent prognostic parameter and is correlated with tumor cell proliferation and apoptosis in malignant melanoma. Int J Cancer 126: 2553-2562.

10. Miura N, Sato R, Tsukamoto T, Shimizu M, Kabashima H, et al. (2009) A noncoding RNA gene on chromosome 10p15.3 may function upstream of hTERT. BMC Mol Biol 10: 5. [Crossref]

11. Miura N, Shimizu M, Shinoda W, Tsuno S, Sato R, et al. (2013) Human RGM249derived small RNAs potentially regulate tumor malignancy. Nucleic Acid Ther 23 332-343.

12. Chen Y, Chen H, Hoffmann A, Cool DR, Diz DI, et al (2006) Adenovirus-mediated small-interference RNA for in vivo silencing of angiotensin AT1a receptors in mouse brain. Hypertension 47: 230-237.

13. O'Neill SM, Hinkle C, Chen SJ, Sandhu A, Hovhannisyan R, et al. (2014) Targeting adipose tissue via systemic gene therapy. Gene Ther 21: 653-661. [Crossref]

14. Askou AL, Pournaras JA, Pihlmann M, Svalgaard JD, Arsenijevic Y, et al. (2012) Reduction of choroidal neovascularization in mice by adeno-associated virus-delivered anti-vascular endothelial growth factor short hairpin RNA. J Gene Med 14: 632-641.

15. Verma UN, Surabhi RM, Schmaltieg A, Becerra C, Gaynor RB (2003) Small interfering RNAs directed against beta-catenin inhibit the in vitro and in vivo growth of colon cancer cells. Clin Cancer Res 9: 1291-1300.

16. Grzelinski M, Urban-Klein B, Martens T, Lamszus K, Bakowsky U, et al. (2006) RNA interference-mediated gene silencing of pleiotrophin through polyethyleniminecomplexed small interfering RNAs in vivo exerts antitumoral effects in glioblastoma xenografts. Hum Gene Ther 17: 751-766.

17. Davis ME, Zuckerman JE, Choi CH, Seligson D, Tolcher A, et al. (2010) Evidence of RNAi in humans from systemically administered siRNA via targeted nanoparticles. Nature 464: 1067-1070.

18. Gomes-da-Silva LC, Fonseca NA, Moura V, Pedroso de Lima MC, Simões S, et al. (2012) Lipid-based nanoparticles for siRNA delivery in cancer therapy: paradigms and challenges. Acc Chem Res 45: 1163-1171.

19. Jo J, Yamamoto M, Matsumoto K, Nakamura T, Tabata Y (2006) Liver targeting of plasmid DNA with a cationized pullulan for tumor suppression. J Nanosci Nanotechnol 6: $2853-2859$.

20. Kushibiki T, Tomoshige R, Iwanaga K, Kakemi M, Tabata Y (2006) In vitro transfection of plasmid DNA by cationized gelatin prepared from different amine compounds. $J$ Biomater Sci Polym Ed 17: 645-658.

21. Mu D, Luan J, Mu L, Xin M (2009) Reoperation for the removal of polyacrylamide hydrogel in the breast: use of periareolar approach under direct visualization. Aesthetic Plast Surg 33: 473-474. [Crossref]

22. Kimura Y, Tabata Y (2010) Controlled release of stromal-cell-derived factor-1 from gelatin hydrogels enhances angiogenesis. J Biomater Sci Polym Ed 21: 37-51. [Crossref]

23. Takeshita F, Patrawala L, Osaki M, Takahashi RU, Yamamoto Y, et al. (2010) Systemic delivery of synthetic microRNA-16 inhibits the growth of metastatic prostate tumors via downregulation of multiple cell-cycle genes. Mol Ther 18:181-187. 
24. Lai EC (2002) Micro RNAs are complementary to 3' UTR sequence motifs that mediate negative post-transcriptional regulation. Nat Genet 30: 363-364. [Crossref]

25. Lewis BP, Shih IH, Jones-Rhoades MW, Bartel DP, Burge CB (2003) Prediction of mammalian microRNA targets. Cell 115: 787-798. [Crossref]

26. Nam JW, Rissland OS (2014) Global analyses of the effect of different cellular contexts on microRNA targeting. Mol Cell 53: 1031-1043. [Crossref]

27. Tsuno S, Wang X, Shomori K, Hasegawa J, Miura N (2014) Hsa-miR-520d induces hepatoma cells to form normal liver tissues via a stemness-mediated process. Sci Rep 4: 3852 .

28. Ishihara Y, Tsuno S, Kuwamoto S, Yamashita T, Endo Y, et al. (2014) Hsa-miR-520d converts fibroblasts into CD105+ populations. Drugs RD 14: 253-264. [Crossref]

29. Ishihara Y, Tsuno S, Kuwamoto S, Yamashita T, Endo Y, et al. (2016) Tumorsuppressive effects of atelocollagen-conjugated hsa-miR-520d-5p on un-differentiated cancer cells in a mouse xenograft model. BMC Cancer 16:415.

30. Abdelkarim M, Guenin E, Sainte-Catherine O, Vintonenko N, Peyri N, et al. (2009) New symmetrically esterified m-bromobenzyl non-aminobisphosphonates inhibited breast cancer growth and metastases. PLoS One 4: e4685. [Crossref]

31. Pereira DM, Rodrigues PM, Borralho PM, Rodrigues CM (2013) Delivering the promise of miRNA cancer therapeutics. Drug Discov Today 18: 282-289. [Crossref]

32. Piao L, Zhang M, Datta J, Xie X, Su T, et al. (2012) Lipid-based nanoparticle delivery of Pre-miR-107 inhibits the tumorigenicity of head and neck squamous cell carcinoma. Mol Ther 20: 1261-1269. [Crossref]

33. Kota J, Chivukula RR, O'Donnell KA, Wentzel EA, Montgomery CL, et al. (2009) Therapeutic microRNA delivery suppresses tumorigenesis in a murine liver cancer model. Cell 137: 1005-1017.

34. Bennett CF, Swayze EE (2010) RNA targeting therapeutics: molecular mechanisms of antisense oligonucleotides as a therapeutic platform. Annu Rev Pharmacol Toxicol 50: $259-293$.

35. Farazi TA, Hoell JI, Morozov P, Tuschl T (2013) MicroRNAs in human cancer. Adv Exp Med Biol 774: 1-20. [Crossref]
36. Li Z, Yang CS, Nakashima K, Rana TM (2011) Small RNA-mediated regulation of iPS cell generation. EMBO J 30: 823-834. [Crossref]

37. Shao W, Paul A, Abbasi S, Chahal PS, Mena JA, et al. (2012) A novel polyethyleneiminecoated adeno-associated virus-like particle formulation for efficient siRNA delivery in breast cancer therapy: preparation and in vitro analysis. Int J Nanomedicine 7: 1575-1586.

38. Nomoto T, Fukushima S, Kumagai M, Machitani K, Arnida, et al. (2014) Three-layered polyplex micelle as a multifunctional nanocarrier platform for light-induced systemic gene transfer. Nat Commun 5: 3545. [Crossref]

39. Kelnar K, Peltier HJ, Leatherbury N, Stoudemire J, Bader AG. (2014) Quantification of therapeutic miRNA mimics in whole blood from nonhuman primates. Anal Chem 86: $1534-1542$

40. Goemans NM, Tulinius M, van den Akker JT, Burm BE, Ekhart PF, et al. (2011) Systemic administration of PRO051 in Duchenne's muscular dystrophy. $N$ Engl J Med 364: 1513-1522. [Crossref]

41. Matsuura K, Murasato K, Kimizuka N (2005) Artificial peptide-nanospheres selfassembled from three-way junctions of beta-sheet-forming peptides. $\mathrm{J} \mathrm{Am} \mathrm{Chem} \mathrm{Soc}$ 127: 10148-10149. [Crossref]

42. Tazawa H, Tsuchiya N, Izumiya M, Nakagama H. (2007) Tumor-suppressive miR34a induces senescence-like growth arrest through modulation of the E2F pathway in human colon cancer cells. Proc Natl Acad Sci USA 104: 15472-15477.

43. Takei Y, Takigahira M, Mihara K, Tarumi Y, Yanagihara K. (2011) The metastasisassociated microRNA miR-516a-3p is a novel therapeutic target for inhibiting peritoneal dissemination of human scirrhous gastric cancer. Cancer Res 71: 1442-1453.

44. Osaki M, Takeshita F, Sugimoto Y, Kosaka N, Yamamoto Y, et al. (2011) MicroRNA-143 regulates human osteosarcoma metastasis by regulating matrix metalloprotease-13 expression. Mol Ther 19: 1123-1130.

45. Hao Z, Fan W, Hao J, Wu X, Zeng GQ, et al. (2016) Efficient delivery of micro RNA to bone-metastatic prostate tumors by using aptamer-conjugated atelocollagen in vitro and in vivo. Drug Deliv 23: 874-881

46. Inaba S, Nagahara S, Makita N, Tarumi Y, Ishimoto T, et al. (2012) Atelocollagenmediated systemic delivery prevents immunostimulatory adverse effects of siRNA in mammals. Mol Ther 20: 356-366.

Copyright: (2019 Ishihara Y. This is an open-access article distributed under the terms of the Creative Commons Attribution License, which permits unrestricted use, distribution, and reproduction in any medium, provided the original author and source are credited. 